\title{
Research on Interactive Art Design Based on Artificial Intelligence
}

\author{
Wang Aili \\ Dalian Polytechnic University, Dalian, Liaoning, China
}

Keywords: AI, Interaction, Art design

\begin{abstract}
The development of AI (Artificial Intelligence) develops from machine perception to pattern recognition based on the original brain cognitive science, and finally from knowledge engineering expert system to new perception and new cognitive logic of knowledge map. AI products continue to create works of art through in-depth study of artists' strokes and according to certain logic, so as to "revive" classic artists once again, thus edifying human sentiment. AI technology is helpful to artistic design and creation. Art designers should combine with AI technology when learning creative programming. With the continuous development of interactive art design, interactive art design works will have wider application fields and development space, and the birth of interactive art design cannot be separated from the application of AI technology. Therefore, it can be said that AI is a creative weapon for interactive artistic design.
\end{abstract}

\section{Introduction}

With the coming of the fourth industrial revolution, AI (Artificial Intelligence), as a major technology, will surely spur the transformation of the whole human society. Looking at the history of mankind and the development of science and technology since modern times, it is not only a history of information revolution, but also a history of energy revolution, and it is also a history of traffic revolution. Information, energy and traffic constitute the three core forces of human science and technology development [1]. In the field of human rational work, AI is revolutionizing many industries, and many mechanized jobs have been replaced by AI. For human beings, AI's participation is already all-round, and this growing thing from human intelligence extends the life cycle and wisdom of human beings as limited living beings, and at the same time, it also embeds a kind of fear in human consciousness [2]. The relationship between AI technology and artistic design has gradually received social attention, so this paper studies the application of AI in artistic design to analyze the relationship between the two.

\section{Artistic Expression of AI}

With the advent of the era of science and technology, the development of AI is very rapid, and it has been accepted that machines have defeated human beings in the chessboard world [3]. From the industrialized society to the information society, the form, scope and space of modern art design are continuously expanding. Every technological and media change endows it with new vitality and connotation, which is one of the symbols of human civilization. There are always some contents in AI that cannot be copied, transcended or replaced. The creativity of human brain represents this part of contents, which is the uniqueness of human beings and the value of species. In recent years, in the fields involved in AI, the combination of art and technology has sublimated to the combination with AI and has received more and more attention [4]. Compared with the time that traditional artistic creation takes months or even years, computer science can help designers finish their creation quickly and efficiently, and mass produce through molds. In contemporary new media art, intelligent painting, design, graffiti and artificial life art are even more references and interactions to science and technology and philosophical concepts. It can also be understood that these arts are expressed by means of scientific and technological media. Being born for use is also the manifestation of human life experience, and more pointing is to the body [5]. Human experience, therefore, has become the most important point to define human and AI in art and design. 
Interactive art is a new artistic form based on the organic combination of computer technology and Internet technology. It reflects one's emotion and artistic concept through the works created, and interacts with works or realizes the embodiment of artistic effect. With the arrival of the new era, our view of things and our attitude towards things should be changed, because there is a close connection between the development of art and the progress of technology. Whether it is professional designers or end-users, there is a phenomenon in the process of visual communication design: images accompanying words that spread, educate and convince audiences will have greater influence. The creativity of human brain can be brought into full play and released in the artistic forms created by human beings. With the innovation of human society, artistic forms are also evolving. AI art and design have reached a certain high level. If designers still stay at the traditional design level, they will be "threatened" by machines [6]. In terms of presentation of works, with the gradual maturity of virtual reality technology, many universities are applying virtual reality technology to the teaching field of art and design majors. Virtual reality can better show design works combining art and technology. AI and human biological intelligence are not incompatible with each other, but exist in what the art world calls "man-machine interaction art", and both blend and cooperate with each other.

\section{Application of AI in Interactive Art Design}

\subsection{In the Field of Intelligent Robots}

With the development of intelligence, intelligent science and technology products represented by artificial intelligent robots are popular. AI can explore logical thinking to solve design problems according to design tasks, such as introducing mental models, pattern judgment and other technologies, and obtaining intelligent decisions through system engineering analysis, synthesis and evaluation. For example, artists can use computer modeling and 3D printing technology to produce products that people need. Can use a large number of color materials in the computer to create more arbitrary works. The difference between the perception of design and art is that the perceived design can be an additional thing, and the concrete "use" of objects mostly follows the needs of human life [7]. For the design of body use, pattern recognition is the key content of computer AI research, that is, through the use of various sensors, the information recognition system is constructed by using information perception technology, and through the simulation of human sensory organs, the system has the general perception ability of human things, thus transforming the physical variables of the research object. Only need to input the design requirements, conditions and related parameters, the system can formulate a lot of design schemes according to the customer's requirements, and can also classify multiple design schemes according to the screening function of AI technology. It has almost become an indispensable part of people's life. From this, we can see that every change in the development of society and science and technology changes the form and expression of traditional artistic design.

\subsection{Interactive Artistic Design of Intelligent Platform}

High-quality art and design rely on hybrid enhanced intelligence technology. AI also plays a role in the field of art creation. With the help of AI, artists' creative thinking can produce very specific artistic works, such as painting works generated by in-depth learning algorithms and music composed by AI. According to the pixel points on the image, a certain algorithm is used to extract the main color distribution area to form a data set, and the color is expressed in the form of characters, and a certain algorithm set is transformed. Re-interpret the photos uploaded by users to generate a photo with artistic flavor. The application method of this software is roughly the same as that of other common software. First, you need to open the software, take photos or upload existing photos, and then select famous painting strokes. And to logo, typesetting, painting, artistic design, illustration, color and electronic equipment for two-dimensional image performance. Using the intelligent picture editing technology, adjust the picture, change the original artistic conception of the picture, and blend in the artistic flavor. Through perception and identification, the position and 
movement speed of the person can be obtained, thus realizing the change of the grass. Different movement directions of the person can lead to different angles of the grass toppling, and different movement speeds of the person can lead to different swaying situations of the grass. For "system" type object design, it involves the common problems of groups, so AI has natural advantages [8].

\subsection{Interactive Artistic Image Processing}

In the interaction design, the initial interaction technology is changed to the interaction process oriented by user's requirements, and the interaction design method will also face innovation. For example, teachers can use multimedia technology to vividly display the contents of classes. Virtual reality technology can make students "truly" place themselves in the works of great artists, breaking the time and place restrictions. Under this definition, it is difficult for AI to completely replace the individual performance of artists and designers in terms of big data and computing operations. The author's thoughts are expressed in visual artistic forms such as pictures or images, which convey some specific information to the conveyed objects and have an impact on the conveyed objects. Therefore, applying AI to art education can help students fully release their thinking and broaden their imagination. It is an embodiment of hypothetical thinking. The works it creates can get feedback in real-time interaction with the audience, which is a dynamic work. Based on the image processing technology of depth learning, through the network neural algorithm, the image information is processed, and the interconnection relationship between a large number of internal nodes is adjusted, so as to achieve the purpose of processing depth information [9].

\section{The Influence of Ai on Interactive Art Design}

\subsection{Innovation of Interactive Design Method in Ai Innovation}

At present, AI is divided into strong AI and weak AI. Strong AI is just like science fiction movies. Machines can communicate with the world like humans. They can learn and remember themselves, reason and solve problems. A good interactive design can enhance the audience's intuitive feelings, improve their participation, make them have a deeper understanding of what the author wants to express, and guide them to make consumption or behavior choices. AI can calculate big data between minutes and seconds, but in the face of people's daily life, such as telling a joke, reciting poetry, singing a music and other events rich in human emotions, the performance of AI is not particularly perfect. Through the application of AI technology, the artistic effect of the work can be further strengthened and the audience and the emotion of the work can be further sublimated. In other words, the user-centered interaction design method should be transformed into a meaningcentered interaction method that takes care of the wisdom of the group. It is through AI's own understanding that it designs a perception that can be obtained by what it considers "human beings"! This is not a wonderful prospect. Contemporary interactive works of art mainly require the common progress of concepts and technologies, and are interwoven with each other. They are indispensable. Artists and designers can create in the form of intelligent technology. New ideas and concepts in creation can also explore technology in turn.

\subsection{The Multi-Dimensional Support for User Experience is Realized}

Emotional computation enables machines to better understand the dialogue intention and deep semantics. Emotion plays an important role in human decision-making, social interaction, perception, memory, learning and creativity. With the integration of intelligent technology, interactivity becomes more important. Creators should not only consider whether the audience is visual or auditory, involving some of the five senses, but also consider having higher interactivity. Through investigation and analysis, it provides college teachers and students with an adaptive learning system. Interaction with teaching materials. For example, in the complete interactive design process, combining with the previous user demand research, combing the conceptual maps in interactive design, creating user models with different demands, and building intelligent interactive interfaces. Through learning AI technology to understand the development of AI, 
teachers' educational and teaching concepts and teaching methods can be changed to lead the future of high-quality education. However, product interaction design is a design method based on technology to make products intelligent. It pays more attention to the interaction process and experience of both parties.

\subsection{Promote the Formation of a New Type of Man-Machine Symbiotic Relationship}

Technological progress not only brings good experience to users, but also enables the public to directly participate in creation, especially interactive works. The public can also become artists. From this point of view, finally, no matter which way AI is led by its self-consciousness, its thinking mode is still the continuation and development of human thinking mode. Therefore, at least the human body experience has been constructed and developed as a part of big data and computation from the beginning. In interactive design, identification of user's needs can optimize the interactive mode and content, realize human-computer, personnel, interpersonal, human environment and other interactions in the interactive process, stimulate innovation consciousness, and promote the formation of collaborative thinking. It not only provides a broad creative space and interactive scheme for the creation of works, but also greatly changes the position of participants in the works and realizes the role change from the information receiver to the controller of works change and the interactive selector of works. With the maturity of video 3D technology and the continuous development of VR, AR and other virtual reality technologies, the original planar visual language has been transformed into panoramic stereoscopic images in a certain way, and the viewing experience is even more shocking. To discuss this issue logically from human thinking, they certainly need art, because the beginning of AI is human's efforts to break through various boundaries.

\section{Conclusion}

AI has a significant impact on the art design industry, such as more convenient and low-cost art learning, which all benefit from AI. The auxiliary tools led by AI make artists' creation more efficient. Art design has always been the product of the development of science and technology. In the new media era, art design expands its content and forms of expression with the progress of science and technology. There are many forms of art, and this form will change with the changes of the times, but the essence of art will not change. Both the artistic form of the work and the aesthetic psychology of the audience are emphasized, and the experience layer and the psychological demand layer are organically integrated. Therefore, the development of art needs a better combination of AI technology and teaching to continuously improve students' aesthetics. With the continuous development of AI technology, the level of interactive art creation and the quality of works will be further improved.

\section{References}

[1] Hu Gang. (2018). From GUI to XUI: Research on "Relationship” Interaction Design in the Age of AI. Art Science and Technology, vol. 031, No. 012, pp. 91-92.

[2] Sun Xiuying. (2018). The Influence of AI on Interaction Design. Art Science and Technology, vol. 031, No. 011, pp. 53.

[3] Yue Cheng. (2019). Development and application of anthropomorphism of AI and cultural design experience. Industrial Design, vol. 000, No. 005, pp. 133-135.

[4] Qin Jingyan. (2017). Research on the influence of AI on interaction design. Packaging Engineering, vol. 038, No. 020, pp. 27-31.

[5] Duan Shaohua. (2017). Application and development of human-computer interaction oriented design in tea store design. Fujian Tea, vol. 039, No. 003, pp. 18-19.

[6] Hui Dan, Wang Qi, Chen Peng, et al. (2019). Application Research of AI Technology in 
Interaction Design. Packaging and Design, No. 3, pp. 118-119.

[7] Zhou Zhiyu. (2019). Research on the ethical issues of AI systems and product design. Creativity and Design, vol. 000, No. 001, pp. 23-30.

[8] Gui Yuhui, Hu Yujia. (2017). Research on the core concepts, educational evolution and practice of interactive design. Design Art Research, vol. 007, No. 002, pp. 93-99,128.

[9] Yi Anan. (2018). The auxiliary role of AI technology in landscape architecture design and construction. Art Education, vol.320, No. 04, pp. 97-98. 